\title{
Prevalence of Tobacco Use and Oral Mucosal Lesions among Nicobarese Tribal Population in Andaman and Nicobar Islands
}

\author{
Anuradha Rajkuwar ${ }^{1}$, Ajay Verma², Hariharavel Vijayapandian ${ }^{3}$, Prafful Kumar ${ }^{4}$, Munish Dheeraj ${ }^{5}$, Vivin Vincent ${ }^{6}$
}

\begin{abstract}
Aim: To determine the prevalence of tobacco use and oral mucosal lesions among Nicobarese tribal populations in Andaman and Nicobar Islands, India.

Materials and methods: Cross-sectional survey and oral examination were done in 400 Nicobari populations in Car Nicobar using the World Health Organization (WHO) format of Oral Health Questionnaire and Assessment forms. The data were then entered and statistical analysis was done using SPSS Inc.; Chicago, Illinois, USA, Version 20.0.

Results: There is high prevalence of tobacco consumption (88.25\%), especially the smokeless form of tobacco. Oral mucosal lesions were present in $25.75 \%$ of the total population, and keratosis was the most prevalent among oral mucosal lesions which was $56.3 \%$.

Conclusion: There is a statistical significance in the presence of oral mucosal lesions who consumes smokeless form of tobacco every day. There is no significance observed between male and female Nicobari tribes.

Clinical significance: This high consumption of smokeless form of tobacco puts both genders in Nicobari tribal population equally at risk of developing precancerous and cancerous lesions. Early intervention and oral health education should be done frequently to prevent the development of oral cancer at early stages in tribal population.

Keywords: Nicobari population, Oral mucosal lesion, Tobacco user, Tribal.

The Journal of Contemporary Dental Practice (2021): 10.5005/jp-journals-10024-3176
\end{abstract}

\section{INTRODUCTION}

The Andaman and Nicobar Islands is an Indian archipelago situated in the Southeastern part of India of the Indian Ocean in the Bay of Bengal. Apart from being a famous tourist destination, it is home for six native tribes of India.' They are the Great Andamanese, Onge, Jarawa, Sentinels, Shompen, and Nicobarese. Among these tribes, Nicobarese and Shompen are restricted to Nicobar group of Islands (Southern part), while the rest are restricted to Andaman group of Islands (Northern part). The Nicobari tribe accounts for over $95 \%$ of the tribal population in the Island. The geographic locations of the Islands are strategically positioned to receive heavy rainfall that favors the dense tropical forest which in turn favors the growth of areca nuts and betel nuts in the Islands. In spite of various tobacco prevention and control policies over the years, it is apparent that the prevalence of tobacco use in India remains moderately intact. Moreover, it has been accounted for a relatively high use of tobacco in provincial than in the urban parts of India. The prevalence of tobacco use in a tribal area is generally highly contrasted with urban and rural partners. ${ }^{2}$ Likewise, Andaman and Nicobar Islands are no exception to the statement.

Addiction to tobacco has been found to be significantly high among the tribal population of India. In Andaman and Nicobar Islands, tobacco chewing is the most common habit of tobacco use and there are different patterns of tobacco chewing found such as use of Zarda Pan, Kagaz Pan, Sookha, Khaini, and Gutkha. ${ }^{3}$ The Nicobarese are found to be fond of smoking tobacco wrapped in the pandanus leaf, cigarette, and bidi. They also follow chewing tobacco with lime and a combination of tobacco and betel leaf which is known as "Nyoop" (betel quid).

Tobacco use is one of the major risk factors for the development of oral mucosal lesions including oral precancer and cancerous lesions. The oral mucosa serves as a protective barrier against

\begin{abstract}
${ }^{1-5}$ Department of Dentistry, Andaman and Nicobar Islands Institute of Medical Sciences (ANIIMS), Port Blair, Andaman and Nicobar Islands, India

${ }^{6}$ Department of Community Medicine, Andaman and Nicobar Islands Institute of Medical Sciences (ANIIMS), Port Blair, Andaman and Nicobar Islands, India

Corresponding Author: Hariharavel Vijayapandian, Department of Dentistry, Andaman and Nicobar Islands Institute of Medical Sciences (ANIIMS), Port Blair, Andaman and Nicobar Islands, India, Phone: +91 9840101393, e-mail:vph.pedodontist@gmail.com

How to cite this article: Rajkuwar A, Verma A, Vijayapandian $\mathrm{H}$, et al. Prevalence of Tobacco Use and Oral Mucosal Lesions among Nicobarese Tribal Population in Andaman and Nicobar Islands. J Contemp Dent Pract 2021;22(9):975-978.
\end{abstract}

Source of support: Nil

Conflict of interest: None

trauma, pathogens, and carcinogenic agents. As a known fact risk factor for the development of oral mucosal changes increases with tobacco use. Oral cancers are found most prevalent $(19.27 \%$ of all cancer cases) among the population of Andaman and Nicobar Islands. ${ }^{3}$

India is home to various tribal communities with widely varying cultures and socioeconomic conditions in different geographic locations. Therefore, a study done in one region of the country cannot be generalized for all tribal communities. While there are a few studies on tribal populations from south and western parts of India, there is a significant literature gap from this union territory. Previous data on oral cancer in the Islands were retrospective study and tobacco use data were based on self-reported rates of tobacco use. They were devoid of oral examination. Hence the present study 
was carried out in Car Nicobar to estimate the prevalence of tobacco practice and oral mucosal lesions in Nicobarese tribal population in the union territory of Andaman and Nicobar Islands in India.

\section{Materials and Methods}

The cross-sectional study design was used to perform the field survey among the Nicobari tribal population. The study was planned to be conducted in Car Nicobar District of the Nicobar Islands. Since Nicobar Islands come under restricted area permit, prior ethical committee clearance, Tribal Council Consent, and Administration approval were obtained. After the approval from the concerned authorities, the survey was carried out by standard operating procedure. Inclusion criteria were that the randomly selected participants of both genders who belong to the Nicobari tribe and above 12 years with consent. Exclusion criteria were other tribal groups Shompen and those who were not willing to give consent.

The survey and examination were done by a single investigator with the help of a translator. The participant's interview was taken in a local language and care was taken to maintain their confidentiality. The oral health status was measured using World Health Organization (WHO) format and data related to the practice of tobacco were collected. Each participant was explained about the study in their native language and informed consent was taken. In case of participants below 18 years of age, consent was taken from parents. Those who were literate were given the questionnaire to fill up on their own. For illiterate participants, the questions were read out and response was marked. For this, the help of an interpreter was taken.

During questionnaire interview (WHO oral health questionnaire total 16 questions) and clinical examination (WHO oral health assessment form), the type of tobacco use, frequency, and oral mucosal lesions were recorded for all the participants. The participants were categorized into four groups according to their tobacco habits such as group A—smokeless form, group B-smoke form, group C - combination of both smoke and smokeless form, and group D-nontobacco habit. Participants with the presence of oral precancerous lesions and conditions were further informed to visit for periodical checkups and treatment for the same. The type of tobacco use, frequency, and oral mucosal lesions were recorded for all the participants as per the WHO format. All the data were entered, and Fisher's exact and Chi-square tests were analyzed using SPSS software version 20 for statistical analysis.

\section{Results}

In the present study, there were 400 Nicobari tribal participants. The mean age is $41.83 \pm 17.5$ years. Out of total study participants, male participants were $38 \%(n=152)$ whereas female participants were $62 \%(n=248)$ (Table 1). Out of the total participants, $83.5 \%$ ( $n=334$ ) were seen with the habit of smokeless form of tobacco, $3.3 \%(n=13)$ were seen with the habit of smoking tobacco, $1.5 \%$ $(n=6)$ with the habit of both smoke and smokeless form tobacco,

Table 1: Percentage of tobacco and nontobacco users among gender

\begin{tabular}{lccc}
\hline & Male & Female & Total \\
\hline No. of participants & $152(38 \%)$ & $248(62 \%)$ & $400(100 \%)$ \\
Tobacco users & $131(32.75 \%)$ & $222(55.5 \%)$ & $353(88.25 \%)$ \\
Smokeless form & 120 & 214 & $334(83.5 \%)$ \\
Smoke form & 7 & 6 & $13(3.3 \%)$ \\
Combination & 4 & & $6(1.5 \%)$ \\
Nontobacco users & $21(5.25 \%)$ & $26(6.5 \%)$ & $47(11.75 \%)$ \\
\hline
\end{tabular}

and $11.8 \%$ ( $n=47)$ were having no tobacco habits. There is high prevalence (88.25\%) of tobacco habits among the tribal population and especially in smokeless form of tobacco.

Table 2 shows that the presence of oral lesions among smokeless form of tobacco consumers was $27.84 \%(n=93)$ whereas oral lesions among tobacco consumers in smoke form were $15.38 \%$ $(n=2)$. It has been observed that participants who were consuming both smokeless and smoke form of tobacco have shown $100 \%$ $(n=6)$ presence of oral lesions. With the absence of tobacco habits, two participants were found to have cleft lip/palate and aphthous ulcer. They contribute $4.25 \%$ of the total oral mucosal lesions. There is statistical significance of 0.0001 (Fisher's exact test $p$ value).

In comparison with the presence of oral lesions among genders (Table 3), 28.2\% among female participants had oral lesions and $21.7 \%$ among male participants had oral lesions. There is no statistical significance observed between male and female participants with respect to the presence of oral lesions.

Table 4 shows among total participants, $61.5 \%(n=246)$ were seen consuming tobacco every day whereas $17.3 \%(n=69)$ consumed several times a week, $4 \%(n=16)$ consumed once a week, $3.8 \%(n=15)$ consumed several times a month, $1.8 \%$ (7) consumed occasionally, and $11.8 \%(n=47)$ never consumed. It is statistically significant with 0.0001 (Fisher's exact test $p$ value).

It is observed that $25.75 \%$ of the total study participants were found with oral mucosal lesions. On comparing different oral mucous lesions found in our study, at the highest rate i.e., $56.3 \%$ of the total mucosal lesions, keratosis is present in the participants. Ulceration was second most common oral lesion with $10.7 \%$, oral cancer (5.82\%), leukoplakia (8.73\%), oral submucous fibrosis $(6.8 \%)$ candidiasis (6.8\%), erythroplakia (1.94\%), lichen planus $(0.97 \%)$, smokers palate (0.97\%), and cleft lip and palate (0.97\%) (Table 5).

\section{Discussion}

Oral mucous membrane is most susceptible to many diseases but astoundingly, very scarce information is available regarding abnormalities of oral mucosa, particularly among the tribal population of India where the use of any form of tobacco products is the most prevalent and major cause of havoc. Car Nicobar Island in the Southern district of Andaman and Nicobar Islands is mostly inhabited by Nicobari aboriginal tribes. Their overall literacy rate is around $71 \%$ and even after 2004 tsunami devastated their life, they still maintain their traditional cultural and social rituals in their daily life. The consumption of smoking and smokeless tobacco varied dramatically in the tribal population in India. Numerous studies have been conducted on the health status of different primitive tribal communities in India but very few studies have been reported on the tribal populace, especially that of Nicobari tribe with regard to oral health. The cross-sectional study was designed to analyze the type of tobacco use, frequency, and oral mucosal lesions in Car Nicobar in participants above 12 years agegroup. This is the first cross-sectional study conducted exclusively involving Nicobari tribe in the union territory of Andaman and Nicobar Islands.

Large consumption of tobacco and areca nut along with geneenvironment interactions increases the risk of oral precancerous and cancerous lesions. ${ }^{4}$ Oral cancer is one of the few cancers whose survival rate has not improved over the years while during the past few decades, a $60 \%$ increase in oral precancerous and cancerous lesions in adults under the age of 40 years has been documented. ${ }^{5,6}$ 
Table 2: Presence of lesion among tobacco habit population

\begin{tabular}{|c|c|c|c|c|c|}
\hline \multirow[b]{2}{*}{ Tobacco habit } & \multirow[b]{2}{*}{ No. of participants } & \multirow[b]{2}{*}{ Percent } & \multicolumn{2}{|c|}{ Lesion status } & \multirow{2}{*}{$\begin{array}{l}\text { Percentage of lesion } \\
\text { occurrence with habit }\end{array}$} \\
\hline & & & Present & Absent & \\
\hline Group A—smokeless form & 334 & $83.5 \%$ & 93 & 241 & $27.84 \%$ \\
\hline Group B-smoke form & 13 & $3.25 \%$ & 2 & 11 & $15.38 \%$ \\
\hline $\begin{array}{l}\text { Group C - combination of smoke and } \\
\text { smokeless form of tobacco }\end{array}$ & 6 & $1.5 \%$ & 6 & 0 & $100 \%$ \\
\hline Group D—no tobacco habit & 47 & $11.75 \%$ & 2 & 45 & $4.25 \%$ \\
\hline Total & 400 & $100 \%$ & 103 & 297 & \\
\hline
\end{tabular}

Fisher's exact test $p$ value $=0.0001$ statistically significant

Table 3: Description of oral lesions among male and female

\begin{tabular}{|c|c|c|c|c|c|}
\hline \multirow[b]{2}{*}{ Sex } & \multirow[b]{2}{*}{ Total } & \multirow[b]{2}{*}{ Percent } & \multicolumn{2}{|c|}{ Lesion } & \multirow{2}{*}{$\begin{array}{c}\text { Percentage } \\
\text { (presence of lesion) }\end{array}$} \\
\hline & & & Absent & Present & \\
\hline Female & 248 & $62 \%$ & 178 & 70 & $28.2 \%$ \\
\hline Male & 152 & $38 \%$ & 119 & 33 & $21.7 \%$ \\
\hline Total & 400 & $100 \%$ & 297 & 103 & \\
\hline
\end{tabular}

Chi-square $p$ value $=0.148$

Table 4: Frequency of tobacco habit

\begin{tabular}{lcccc}
\hline & & & \multicolumn{2}{c}{ Lesion code } \\
\cline { 3 - 5 } Duration & Frequency & Percent & No & Yes \\
\hline 1-never & 47 & $11.8 \%$ & 45 & 2 \\
2-seldom & 7 & $1.8 \%$ & 7 & 0 \\
3-several times & 15 & $3.8 \%$ & 13 & 2 \\
a month & & & 14 & 2 \\
4-once a week & 16 & $4.0 \%$ & 14 & \\
5-several times & 69 & $17.3 \%$ & 63 & 6 \\
a week & & & & \\
6-every day & 246 & $61.5 \%$ & 155 & 91 \\
Total & 400 & $100 \%$ & 297 & 103 \\
\hline
\end{tabular}

Fisher's exact test $p$ value $=0.0001$

Table 5: Distribution of oral lesion among different tobacco habits

\begin{tabular}{lcc}
\hline Oral lesions & Overall & Percentage \\
\hline Oral cancer & 6 & $5.82 \%$ \\
Leukoplakia & 9 & $8.73 \%$ \\
Lichen planus & 1 & $0.97 \%$ \\
Ulceration & 11 & $10.7 \%$ \\
Erythroplakia & 2 & $1.94 \%$ \\
Candidiasis & 7 & $6.8 \%$ \\
Oral submucous fibrosis & 7 & $6.8 \%$ \\
Keratosis & 58 & $56.3 \%$ \\
Cleft lip and palate & 1 & $0.97 \%$ \\
Smokers palate & 1 & $0.97 \%$ \\
Total & 103 & $100 \%$ \\
\hline
\end{tabular}

The present study highlights relatively high prevalence of tobacco consumption habits in the Nicobarese tribal population. Overall $88.3 \%$ of participants were tobacco users whereas $11.7 \%$ were nontobacco users. This percentage is relatively higher in the present study compared to the study done by Manimunda et al. ${ }^{1}$ in which the tobacco consumption in Nicobari was $83.9 \%$. Similarly,
Vijayakumar et al. ${ }^{7}$ found $80 \%$ in Sugali tribes in Telangana region, Chellappa et al. ${ }^{8}$ found $64.55 \%$ adults among tribal gypsies in Thoothukudi district, Anjali et al. ${ }^{9}$ showed $43.8 \%$ had tobacco habits in Nilambur forest, Kerala. Verma et al. ${ }^{10}$ found a $43.38 \%$ prevalence of tobacco consumption in tribal districts of Madhya Pradesh whereas Kumar et al. ${ }^{11}$ found $45 \%$ in Jharkhand.

Tribal populations in India, similar to other underprivileged groups, have their rational number of distress, and many times they are hidden and unnoticed from the "mainstream" India. Diseased state and malnourishment are more common among indigenous population in comparison with the average Indian population. Currently, $28.6 \%$ of the population uses tobacco products in India. ${ }^{12}$ The difference could be seen because the factors that impact the tobacco consumption were present in the local environment predominantly and also pretentious by beliefs and behaviors toward tobacco habits.

Smokeless tobacco use is documented in many countries. Oral use of smokeless tobacco is ubiquitous; India has major number of smokeless tobacco users in the world and there has been substantial increase in smokeless tobacco use in all age groups. In the present study, frequency of tobacco consumption was observed in $61.5 \%$ of participants on a daily basis. In type of tobacco use $83.5 \%$ of Nicobarese were using smokeless form of tobacco, smoke form in $3.3 \%$ and combination users of both smoke and smokeless form of tobacco were $1.5 \%$. In a study conducted by Chellappa et al., ${ }^{8} 63.4 \%$ were using smokeless tobacco, $29.1 \%$ were using smoke form, and $7.5 \%$ were using both. Manimunda ${ }^{1}$ found that $40.9 \%$ were using smokeless tobacco, $1.7 \%$ were using smoking form, and $6.3 \%$ using both forms of tobacco.

In the present study, there is no significant difference between male and female participants in the overall tobacco consumption habit, which suggests that both genders are equally at risk of developing precancerous and cancerous lesions. Whereas a study reported by Chellapa et al. ${ }^{8}$ shows that tobacco usage was more among female participants than males and Verma et al. ${ }^{10}$ observed that $66.9 \%$ of males had the habit of tobacco, which is nearly double as compared to $33.11 \%$ in females. In the present study, it was also observed that smokeless form of tobacco habit was practiced more among females and the use of combination tobacco was practiced more in the males.

The prevalence of oral mucosal lesions in the present study was found to be $25.8 \%$. Similarly, Patil et al. ${ }^{13}$ reported $26.8 \%$ prevalence of oral mucosal changes in subjects with habits, while it is lower than the $36.36 \%$ prevalence reported by Verma et al. ${ }^{10}$ and $39.1 \%$ by Kamble et al. ${ }^{14}$ In the present study, the most common oral mucosal lesion observed was keratosis which was $60.2 \%$. The development of these oral lesions majorly depends on early onset 
of habit, frequency, type, form of tobacco use, amount of daily use, and site where it is frequently placed. ${ }^{15}$

The second most common lesion observed in the Nicobari tribal population was ulceration, which was $10.7 \%$ followed by rest of the oral lesions. High occurrence of ulceration may be attributed to the fact that Indian population apply lime in the smokeless form of tobacco and consume spicy foods which may enhance the degradation of oral mucosa or result in acidic regurgitation leading to ulcers. Ulcerations may be result of stress, trauma from teeth, and dietary deficiency of vitamins. Accordingly, our study conducted by Abdul Shahid et al. ${ }^{3}$ reported that oral cancer was the most common cancer which comprised $19.61 \%$ of all the cancer cases in Andaman and Nicobar Islands and in which 5.09\% were reported from Nicobar District.

No significant difference was observed between male and female participants in the presence of lesion which is in accordance with the overall tobacco consumption habit, which suggests that both genders are equally at risk of developing precancerous and cancerous lesions.

In the present study, habit of combined smoke and smokeless form of tobacco showed $100 \%$ development of oral lesions. This could be due to adverse effects of nicotine, tar content in smoke form, and different smokeless forms, such as areca nuts, dry mixture of powdered tobacco, slaked lime, and areca nut flakes, which are chewed or sucked orally. Due to placement of smokeless form in the oral cavity, which may increase, the concentration of carcinogens and the failure to clean the carcinogens from the surface augment this effect. All the tobacco forms with higher frequency and for longer duration in contact with oral mucosa lead to the development of oral mucosal lesions. In combination with tobacco habit, the risk is increased twice, which leads to the higher development of oral precancerous lesions and conditions.

Even though $61.5 \%$ of participants were seen consuming tobacco every day, the prevalence of oral mucosal lesions in the present study is less compared to other studies. This could be due to the use of different tobacco forms, quality, and quantity. Tribals widely use locally available areca nuts, betel leaves, and tobacco as compared to the company processed products compared with other states of India.

Limitations of the present study rose when strict COVID protocols were implemented due to spread of coronaviruses. People were reluctant and in fear of participating in the study since it involve oral examination. The additional information on the questionnaire has not been revealed since we received administrative approval on tobacco habits and oral lesions alone. In future, the comprehensive study can be done in all other tribal communities in Andaman and Nicobar Island with the result and effect of the present study.

\section{Conclusion}

With very little information available regarding oral health status among Nicobari tribal population in India, the prevalence of tobacco consumption, which revealed to be higher among both males and females especially smokeless form of tobacco, puts both genders are equally at risk of developing precancerous and cancerous lesions. The overall prevalence of tobacco consumption at $88.25 \%$ and oral mucosal lesions being at $25.8 \%$, immediate oral health education and camps should be conducted frequently for the betterment of tribal population.

\section{ACKnOWLedgments}

Andaman and Nicobar Administration, Port Blair. Tribal Council, Car Nicobar, Andaman and Nicobar Islands. Department of Education, Car Nicobar.

\section{References}

1. Manimunda SP, Benegal V, Sugunan AP, et al. Tobacco use and nicotine dependency in a cross-sectional representative sample of 18,018 individuals in Andaman and Nicobar Islands, India. BMC Public Health 2012;12(1):515. DOI: 10.1186/1471-2458-12-515.

2. Rahul GN. Tribal tobacco survey among young adolescent of central part of India. World J Dent 2019;10(2):109-113. DOI: 10.5005/ jp-journals-10015-1614.

3. Abdul Shahid PP, Pooja Gogia B, Nagma R. Comprehensive analysis of cancer burden in Andaman and Nicobar islands: a descriptive study. Int J Contemp Med Res 2017;4(2):357-360. Available from : https://doi. org/10.21276/ijcmr

4. Khanna S. The interaction between tobacco use and oral health among tribes in central India. Tob Induc Dis 2012;10(1):16. DOI: 10.1186/1617-9625-10-16.

5. Lane PM, Gillhuly J, Whitehead P, et al. Single device for the direct visualisation of oral cavity tissue fluorescence. J Biomed Opt 2006,11(2):024006. DOI: 10.1117/1.2193157.

6. Myers LN, Elkins T, Roberts D, et al. Squamous cell carcinoma of the tongue in young adults: increasing incidence and factors that predict treatment outcomes. Otolaryngol Head Neck 2000;122(1):44-51. DOI: 10.1016/S0194-5998(00)70142-2.

7. Vijayakumar N, Rohini C, Reddy C, et al. Assessment of oral health status and treatment needs among Sugali Tribes in Telangana region: a cross-sectional study. Int J Oral Health Med Res 2017;3(6):21-26. Available from: https://doi.org/10.38110/ijohmr

8. Chellappa LR, Leelavathi L, Indiran MA, et al. Prevalence and dependency of tobacco use among tribal gypsies in Thoothukudi district - a cross sectional study. J Family Med Prim Care 2021;10(2):738744. DOI: 10.4103/jfmpc.jfmpc_1344_20.

9. Anjali S, Shivakumar M, Ranganath $S$, et al. Assessment and comparison of tobacco dependence level among Cholanaicken and Kattunaicken tribal groups of Nilambur Forest, Kerala: a questionnaire study. J Indian Acad Dent Spec Res 2017;4(2):42-45. DOI: 10.4103/ jiadsr.jiadsr_14_17.

10. Verma P, Saklecha D, Akshar P. A study on prevalence of tobacco consumption in tribal district of Madhya Pradesh. International Journal of Community Medicine and Public Health 2018;5(1):76-80. DOI: 10.18203/2394-6040.ijcmph20175508.

11. Kumar G, Dileep CL, Sethi AK, et al. The Birhor tribes of Ramgarh District, Jharkhand - a ferret into their oral health status and treatment needs. Med Pharm Rep 2019;92(2):178-184. DOI: 10.15386/ cjmed-1025.

12. Rai $B, B r a h m a n k a r M$. Tobacco use among Indian states: key findings from the latest demographic health survey 2019-2020. Tob Prev Cessat 2021;7:19. DOI: 10.18332/tpc/132466.

13. Patil PB, Bathi R, Chaudhari S. Prevalence of oral mucosal lesions in dental patients with tobacco smoking, chewing, and mixed habits: a cross-sectional study in South India. J Fam Community Med 2013;20(2):130-135. DOI: 10.4103/2230-8229.114777.

14. Kamble KA, Guddad SS, Nayak AG, et al. Prevalence of oral mucosal lesions in Western Maharashtra: a prospective study. J Indian Acad Oral Med Radiol 2017;29(4):282-287. DOI: 10.4103/jiaomr. JIAOMR_14_17.

15. Sahitha R. Effects of smokeless tobacco, betel quid and areca nut on oral mucosa. IOSR J Dent Med Sci 2014;13(2):8-11. DOI: 10.9790/085313210811. 